The S. F. Smerd Memorial Lecture

The Sun of Stefan Smerd

J. P. Wild

CSIRO, Dickson, A.C.T. 2602, Australia

This lecture commemorates the memory, so recent, of our esteemed and much-loved colleague, Steve Smerd, who died in Sydney a few days before Christmas day last year. The fact that there was a spontaneous move to pay our respects in this unusual way, dedicating an international scientific symposium to honour his memory, must mean that we are dealing with a special individual as a scientist. What do we remember about Steve, the scientist, that was special? I do not think it will be especially for his writings and publications. Although these included some that were definitive and highly significant, they were rather few in number. Although he has so much to tell Steve was not a good communicator through the written word. Though strictly logical, in a teutonic sort of way, his sentences had the tendency of never quite terminating, and their comprehension sometimes demanded of the reader the same kind of determined concentration as is required in the solution of a second order differential equation; he needed, and usually has, a co-author or a helper. To his Sydney-based colleagues by far his most famous writings were universally known as 'the unpublished works of S.F. Smerd' these, the mightly efforts that never quite came to the public eye, were voluminous indeed. No, Steve's method of communication was not through writing, but rather through a kind of oral evangelism. Paul Simon of Meudon Observatory, himself the holder of holy orders, once told me that a visit from Steve was like a visit from the Pope himself. It was not the fact that Steve was sometimes inclined to pontificate, but rather that, though a modest and self-effacing man, he spoke with such authority and omniscient confidence about everything that one was left in no doubt that here indeed was the true word spoken by the priest from on high.

Steve had many interests. I think always of his intense interest in politics which I described on another, more solemn occasion, as lying somewhere between old-fashioned liberalism and old-fashioned socialism. But his abiding interest above all else was the central point of his profession - the Sun. And in talking of the Sun and its basic understanding he interacted marvellously with his colleagues the world over. That is what makes him so special. He was always close to the observa-

M.R. Kundu and T.E. Gergely (eds.), Radio Physics of the Sun, 5-21.

Copyright $\odot 1980$ by the IAU. 
tional data (as Sydney-based people have to be), yet his special forte was interacting with theorists: stimulating them, getting them interested, informing them, bleeding them of what they know. People like Westfold, Twiss, Melrose, Zheleznyakov, Takakura, Goldman, Grognard, Dean Smith and many others. If you had to describe his greatest value in one word it would be as a catalyst. Our solar group was fortunate indeed to own him and his contributions go far beyond the works that bear his name. It is fitting therefore that in this memoral lecture I too go beyond his own personal work and cover fields that put his personal work in perspective, including the work in which he had day-to-day contact and over which he had day-to-day influence.

We should begin therefore with the beginnings of solar radio astronomy in Australia, a year or two before Steve came onto the scene.

The Beginnings

By the end of the war Hey, at metre wavelengths, and Southworth, at centimetre wavelengths, had discovered the Sun's radio emission. Meanwhile, in Sydney the CSIRO Division of Radiophysics, which had been preoccupied for 6 years with military radar, sought few fields of research appropriate to peace time. Joe Pawsey, later to become the father figure of Australian radio astronomy, learned of the metre wavelength solar emission through a New Zealand report, and immediately sought to detect the emissions using military radar receivers north and south of Sydney Harbour. The Sun was first detected on 3 October, 1945.

The radiation was highly variable and found to correlate with sunspot number. A few months later, using the sea as the mirror in a Lloyd's mirror interferometer and with the fortuitous aid of a giant sunspot group, Pawsey and his colleagues demonstrated that the emissions came from the vicinity of sunspots. Meanwhile much attention was being given to the question of accurate calibration of the receiving equipment and the quantification of the radiation. This led to Pawsey's concept of the "apparent temperature", $\mathrm{T}_{\mathrm{a}}$, of the solar disk. Pawsey noticed that whilst on many days there were readings of $\mathrm{T}_{\mathrm{a}}$ of hundreds or thousands of millions of degrees Kelvin, the value at metre wavelengths never went below about $10^{6} \mathrm{~K}$. He discussed this result with the eminent ionospherist, Dr. D. F. Martyn, the first (1939) Chief of Radiophysics but then seconded from Radiophysics to Mount Stromlo Observatory (near Canberra). Martyn, intimately familiar with the behaviour of radio waves in an ionized gas, soon put two and two together. With the aid of available knowledge of the Sun he concluded that metre waves originated in the Corona, centimetre waves in the chromosphere and that Pawsey's million degree minimum was in fact a measure of the coronal temperature, in confirmation of Edlen's 1942 conclusion from optical evidence. He worked out a first order theory of the radiation from what he called the 'Quiet Sun' which he published first in Nature and later in the Proceedings of the Royal Society. In the process however he ruffled a few feathers in Radiophysics because his theory was presented, solo, as a prediction rather than as a piece of collaborative work with Pawsey. The later was 
thus put in a position of hastily despatching a note to Nature on the observational results; it was typical of Pawsey's modesty that he presented these as confirming Martyn's 'prediction'. Unbeknown to the actors of this drama, a young physicist in the Soviet Union named V. L. Ginsburg was coming to conclusions similar to those of Martyn. Thus it is to these two physicists that we owe our first perception of the nature of one component of the radio emissions of the Sun.

The Quiet Sun

Like a number of other physicists, including Sir Hermann Bondi, S. F. Smerd had spent almost the entire war surrounded by barbed wire. To use Bondi's words: for the first half of the war the barbed wire was to stop him from getting out; and the second half to stop other people getting in. For in 1940 Smerd, who had escaped to Britain from his native Austria soon after Hitler moved in, was routinely interned as an enemy alien. Later after his release he was engaged in naval radar research at the high-security Admiralty Signal Establishment at Whitley. At the end of the war he emigrated to Australia and joined Radiophysics in May 1946. There he teamed with K. C. Westfold to form the theoretical section of Pawsey's new 'solar noise' group. They worked well together: Steve the physicist and tremendous gatherer, assimilator and dispenser of knowledge; Westfold the highly competent and innovative applied mathematician.

They set to work developing a proper theory of the quiet Sun to replace the rough and ready theory of Martyn (which Westfold, especially, found quite unsatisfactory). They argued that the only way to arrive at a proper theory was first to determine the path along which each 'ray' travelled; this was worked out by Westfold for a spherically symmetrical atmosphere on the basis of Snell's law using the appropriate form for the refractive index. The next step was to determine, for each element, ds, along' this path, how much radiation was emitted and how much was absorbed; and hence to find the emergent intensity. Thus, it was necessary to adapt the equation of transfer, which Eddington and Milne has used to study the interior of stars, to the very different medium of a fully ionized atmosphere. They needed therefore to incorporate the theory of radiation in an ionized gas, as given by Lorentz or (when magnetic fields are taken into account), by Appleton and Hartree. In the process they found mistakes in the accepted literature which needed correction.

They thus established the now well-known formulae for the intensity of a ray emerging from a spherically symmetrical atmosphere. In a slightly updated and simplified form (defining optical depth relative to the observer rather than the far end of the ray), they gave as the emergent intensity of a ray measured to the point where the optical depth is $\tau$,

$$
I=\int_{0}^{\infty}\left(n / \kappa \mu^{2}\right) e^{-\tau} d \tau
$$


where the optical depth $\tau$ is given by

$$
\tau(s)=\int_{s}^{\infty} \kappa d s,
$$

$\mu$ is the refractive index and $\kappa$ and $n$ denote the absorption coefficient and emissivity. They showed that if, and only if, the distribution of velocity is Maxwellian appropriate to the temperature $\mathrm{T}$ and $\mathrm{h} \nu<<\mathrm{kT}$, equation (1) reduces to

$$
\mathrm{T}_{\mathrm{B}}=\int_{0}^{\infty} \mathrm{T} \mathrm{e}^{-\tau} \mathrm{d} \tau \text {. }
$$

Smerd and Westfold showed how to calculate all the necessary quantities $\kappa, \eta$ and $\mu$ for the solar atmosphere and derived the formula for calculating $\tau$ for a speherically symmetrical atmosphere viz.

$$
\tau=\frac{R_{\omega}}{\nu^{2} c} \int_{\rho}^{\infty} \frac{\nu_{c} \nu_{p}^{2} d \rho}{\sqrt{\mu^{2}-d^{2} \rho^{2}}},
$$

where $\nu, \nu_{c}$ and $\nu_{p}$ are the frequency, the collision frequency and (what is now called) the plasma frequency respectively, $\rho$ is the radial coordinate with solar centre as pole, and $d$ the projected distance of the emergent ray from the centre of the solar disk (see Figure 1). $\rho$ and $d$ are measured in units of the solar radius $R_{0}$.

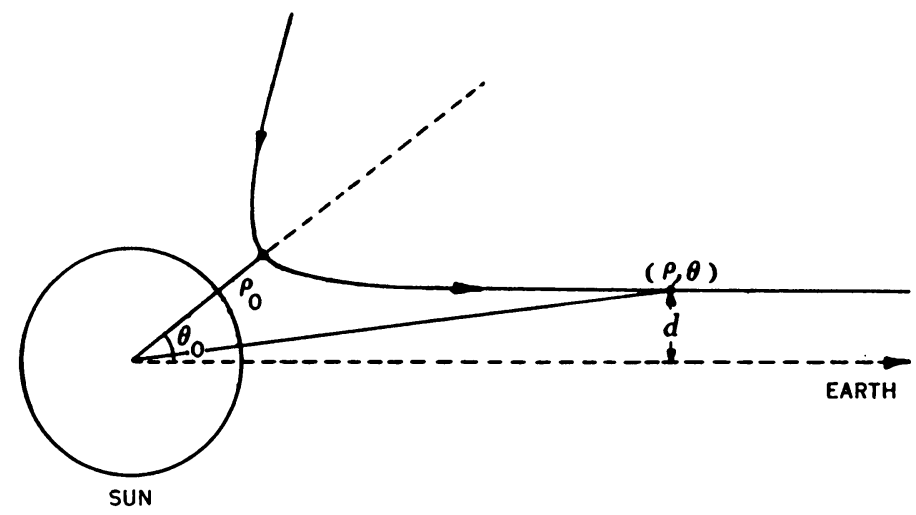

Fig. 1. The trajectory of a ray through a spherically symmetrical atmossphere. 
The quantities $k$ and $\eta$ were derived on the assumption that the radiation process was due to the hyperbolic encounters between electrons and protons in the ionized gas - free-free transitions or bremsstrahlung.

The basic theory of this work was published in a paper by Smerd and Westfold (1949) and its detailed application to the quiet Sun in a paper by Smerd (1950a). These papers are classics and remain to this day definitive works.

Smerd's paper involved an enormous amount of computation. There were no electronic computers around in those days and it all had to be done by hand - he spent hours each day turning the handle of a Brunswigger calculator (e.g. to multiply by 7 you turned once forward in the ten's place, then shifted to the units and turned 3 back). Sharing a room with Steve in those days was listening to the gear meshings of a Brunswigger (see Figure 2). His computations predicted, among many other

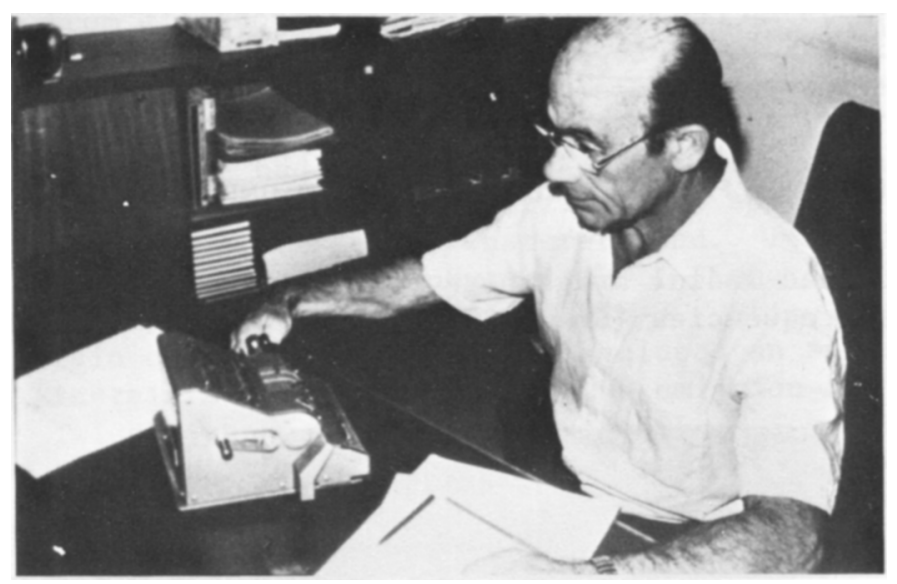

Figure 2. Smerd with his Brunswigger calculator.

things, that at $1 \mathrm{~cm}$ wavelength the disk brightness distribution should be rectangular, at microwaves it should show marked limb brightening, whilst at metre wavelengths it should be bell-shaped (see Figure 3). All these predictions have been subsequently confirmed by observations.

Many years later a remarkable event was recorded at $80 \mathrm{MHz}$ with the Culgoora Radioheliograph (see Figure 4) in which half the Corona could be seen illuminated from behind by a flare event originating on the invisible hemisphere of the Sun. This provided a dramatic demonstration of the opacity of the Corona to radio waves. It was highly appropriate that the event was recorded and analysed by Smerd (1970).

By 1950 Steve had become the repository of a mass of information 


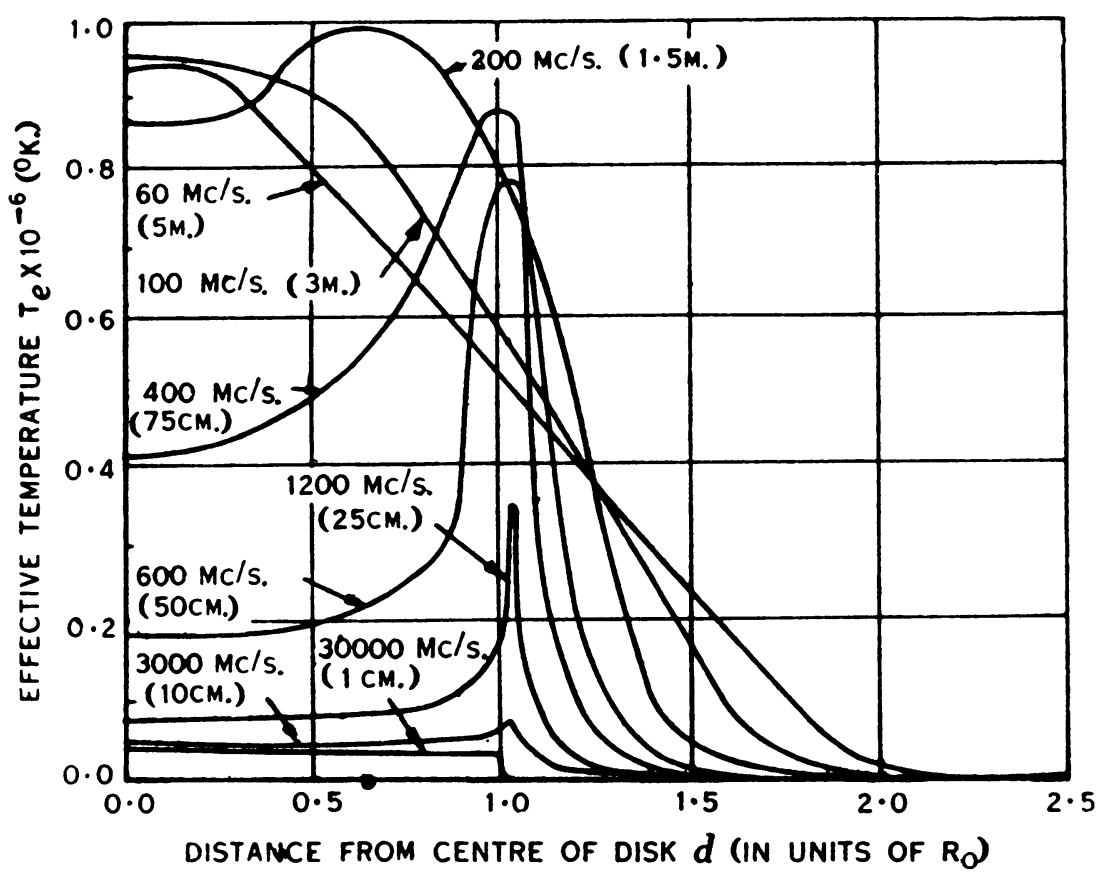

Figure 3. Predicted radial brightness distribution over the solar disk at various frequencies (Smerd 1950a).

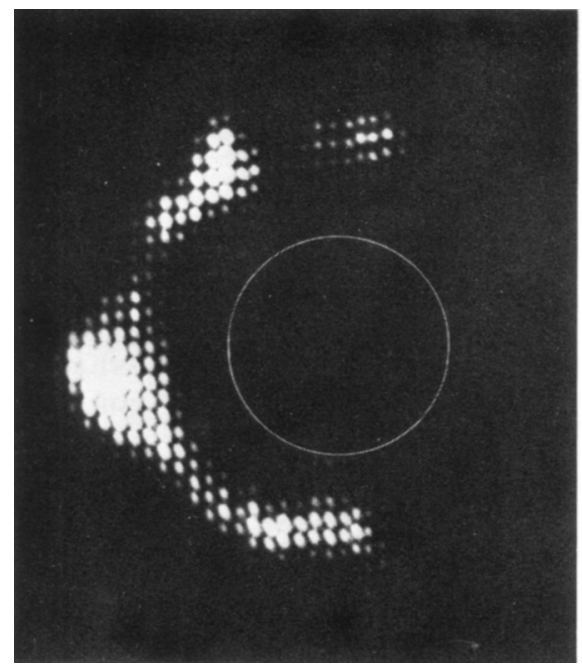

Figure 4. $80 \mathrm{MHz}$ radioheliogram showing outburst from invisible hemisphere of Sun occulted by the solar corona (Smerd 1970). 
about the Sun. Pawsey called him a 'walking encyclopaedia' on solar matters. It seemed desirable that as much as possible of this information should be made known to the world. So in the same year he published (Smerd 1950b) an assemblage of data which was to become the radio astronomers' indispensable reference to the solar atmosphere. It was entitled 'A radio frequency representation of the solar atmosphere'. This was often known locally as 'A wadio-fwequency Wepwesentation...' in view of Steve's difficulty in getting his tongue round the eighteenth letter of the alphabet. Of even more significance in the quest to expose Steve's knowledge of his subject was the chapter 'Solar Radio Emission' which he wrote jointly with Joe Pawsey for Kuiper's celebrated book 'The Sun'. This review (Pawsey and Smerd 1953) is again a classic and I could advise no better introduction to the subject for a student of the 1980's.

Generalization to Non-thermal Radiation

During the 1950 's the generation of non-thermal radiation in bursts became an exciting field of study. Apart from collective processes, which we consider later, most attention was given to the gyromagnetic radiation from electrons in a magnetic field. Later came the suggestion of Alfvén and Herlofson that synchotron radiation could be important in radio astronomy, a hypothesis first applied to the Sun by Boischot and Denisse. Meanwhile Smerd, conditioned by the rigour of the technique that he and Westfold had developed to explain the solar bremsstrahlung began searching for ways of generalizing this approach to take account of all microscopic processes of whatever kind. I beleive a critical step forward came when the English physicist Dr. Richard Twiss spent an extended period in Radiophysics around 1956-7. Stimulated by the search for the origin of burst radiation he developed an entirely new approach based on Einstein's quantum theory of the emission and absorption of radiation. Twiss's paper was published in 1958. Few people comprehended it at the time and it passed largely unnoticed. But not so with Smerd who saw in it the possible answer to a generalized theory of emission.

When a few years later we were co-authors of a review article (Wild, Smerd and Weiss 1963), Smerd proposed that we should present the subject of emission mechanisms as a generalization of equation (3) using Twiss's approach to effect the generalization. This looked increasingly promising the more we looked at it. He and I worked together on the project he provided all the physical input, while I translated his notions into English and worked out the mathematical applications to various processes Our collaboration, normally complementary, was in this instance exceedingly close, closer than at any other time. We were able to extend and considerably simplify Twiss's concept, mainly through the recognition that equation (6), below, was of general validity because of the principle of detailed balancing. The argument (simplified to the iostropic case) runs as follows:-

The general solution of the equation of transfer, irrespective of emission or absorption process, is given by equations (1) and (2). Thus if general expressions for $k$ and $n$ can be devised a general solution for the intensity is obtained. 
Consider a centimetre cube within the source region and a ray passing through it normal to one pair of surfaces. Suppose the particles in the cube are capable of assuming only two energy states ' 1 ' and ' 2 ', and that the number of particles in each state is $n_{1}$ and $n_{2}$ respectively. Then in terms of the Einstein coefficients $A_{21}, B_{21}$ and $B_{12}$, the net amount of radiation absorbed over the unit distance is $I\left(n_{1} B_{12}-n_{2} B_{2 l}\right) h v$ so that the absorption coefficient is

$$
\kappa=\frac{1}{I} \frac{d I}{d s}=\left(n_{1} B_{12}-n_{2} B_{21}\right) h \nu
$$

Likewise the coefficient of emission is

$$
n=n_{2} A_{2 l} h \nu \text {. }
$$

The Einstein theory gives the relationships between the coefficients as

$$
\begin{aligned}
& \frac{A_{21}}{B_{21}}=\frac{\mu^{2} h \nu^{3}}{c^{2}}, \\
& \frac{B_{21}}{B_{12}}=\frac{g_{1}}{g_{2}} .
\end{aligned}
$$

where the g's denote the statistical weights of the states. Whence equations (4) to (7) give

$$
\kappa=\frac{c^{2}}{\mu^{2} v^{2}}\left(\frac{n_{1}}{g_{1}}-\frac{n_{2}}{g_{2}}\right) A_{21} g_{1}
$$

We now consider the classical case in which there is a continuum of energy levels $\varepsilon$, so that transitions giving rise to emission of frequency h $\nu$ are those between $\varepsilon$ (level ' 2 ') and $\varepsilon$-hv (level ' 1 '), where it is stipulated that $\mathrm{h} \nu \ll \varepsilon$, and integration over all $\varepsilon$ is necessary. Thus the symbols with suffixes 1 and 2 are now replaced by continuous functions of $\varepsilon$ as follows:

$$
\begin{array}{ll}
\mathrm{A}_{21} \mathrm{h \nu} & \rightarrow \mathrm{Q}(\varepsilon), \\
\mathrm{n}_{1} & \rightarrow \mathrm{n}(\varepsilon) \mathrm{d} \varepsilon, \\
\frac{\mathrm{n}_{1}}{\mathrm{~g}_{1}} & \rightarrow \frac{\mathrm{n}(\varepsilon)}{\mathrm{g}(\varepsilon)}=\mathrm{NF}(\varepsilon), \\
\frac{\mathrm{n}_{1}}{\mathrm{~g}_{1}}-\frac{\mathrm{n}_{2}}{\mathrm{~g}_{2}} & \rightarrow \mathrm{N} \frac{\mathrm{dF}}{\mathrm{d} \varepsilon} \mathrm{h} \nu .
\end{array}
$$


Here $Q(\varepsilon)$ is the emissivity of a single particle (electrons in our case) by whatever microscopic emission process is specified; $N$ is the total number of electrons; the function $F(\varepsilon)$ as defined by equation (11) is recognized from classical statistical mechanics as the a priori probability distribution of energy in momentum space (e.g. in the case of thermodynamic equilibrium $F(\varepsilon)$ is the exponential distribution of Boltzmann). Substituting equations (9) to (12) in equations (5) and (8) and integrating over $\varepsilon$ we find

$$
\begin{aligned}
& \eta=N \int_{0}^{\infty} F(\varepsilon) Q(\varepsilon) g(\varepsilon) d \varepsilon, \\
& \kappa=-\frac{c^{2} N}{\mu^{2} \nu^{2}} \int_{0}^{\infty} \frac{d F}{d \varepsilon} Q(\varepsilon) g(\varepsilon) d \varepsilon .
\end{aligned}
$$

An alternative form of equation (14) is obtained by integrating by parts (one term vanishing in practice), thus

$$
\kappa=\frac{c^{2} N}{\mu^{2} \nu^{2}} \int_{0}^{\infty} F(\varepsilon) \frac{d}{d \varepsilon}\{Q(\varepsilon) g(\varepsilon)\} d \varepsilon .
$$

Here then was the generalization - and a surprisingly elegant one that Smerd had been seeking. It showed clearly the different roles played by the a priori energy distribution of the electrons $F(\varepsilon)$ and the electron emissivity $Q(\varepsilon)$. It is easily verified that when $F(\varepsilon)$ is the Boltzmann distribution (thermodynamic equilibrium) and hv $<\mathrm{kT}$ one arrives at equation (3) irrespective of the emission process.

The application of these results to different processes - bremsstrahlung, gyro and synchrotron processes - is given in the original paper (here we extended, and sometimes differed from, the original conclusions of Twiss). Of special interest was the qualitative conclusion that followed at once from equations (14) and (15): that under certain circumstances the absorption coefficient could be negative - i.e. amplification occurred as in a maser. Either of two conditions are necesary: either $\mathrm{dF} / \mathrm{d} \varepsilon$ must be positive - indicating overpopulation of higher energy states in some part of the energy distribution; or

$$
\frac{d}{d \varepsilon}\{Q(\varepsilon) g(\varepsilon)\}
$$

must be negative - as happens on the rise to a spectral peak in some part of the emissivity spectrum (see Figure 5). We confirmed Twiss's result that the absorption coefficient of gyro radiation could be negative but denied his result that that of synchronotron radiation could also. 

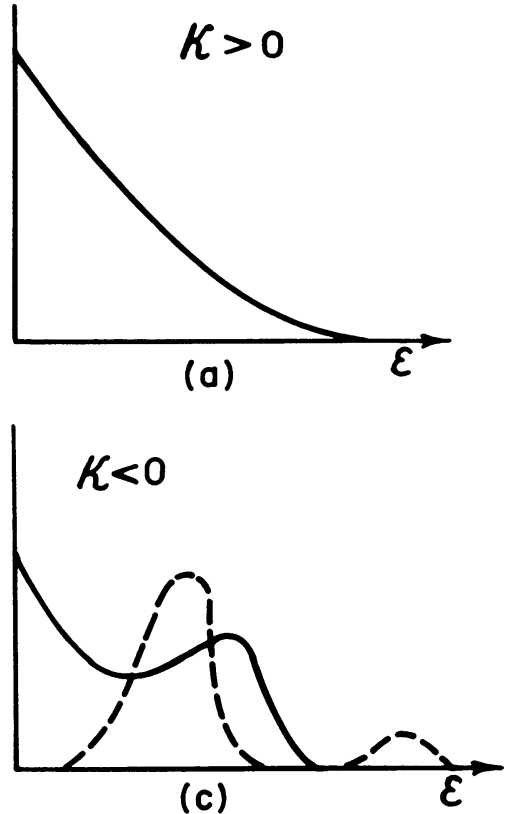

$F(\varepsilon)$
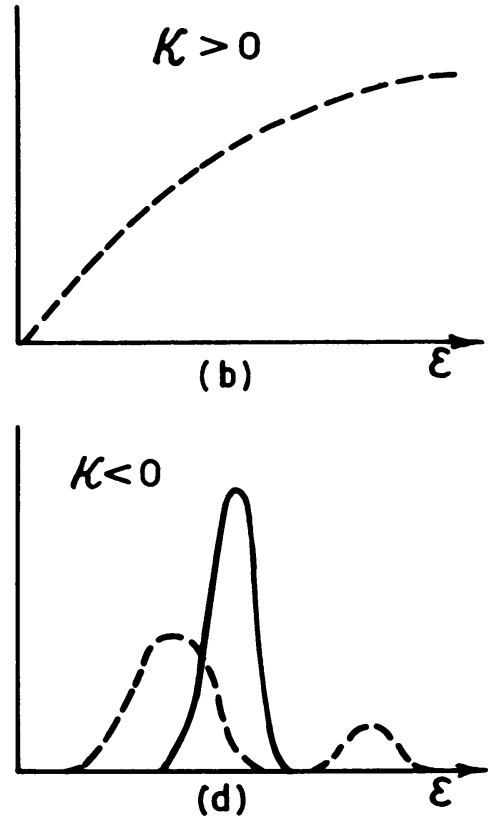

$g(\varepsilon) Q(\varepsilon)$

Figure 5. Conditions for positive and negative absorption coefficient, K. (Wild, Smerd and Weiss 1963).

Type III Bursts

In order to introduce another aspect of this topic, I now return to the late 1940's, soon after Smerd's arrival in Australia. I had joined Radiophysics in February 1947 and a year later, much to my delight, I became a member of the solar noise group and was permitted the task of trying to build a solar spectrograph. I was fortunate in sharing a room with Smerd and Westfold; it was an education and an entertainment, of enduring benefit to me. The spectrograph actually worked (much to most people's surprise). With the aid of my trusted technical assistant, Bill Rowe, there followed four exciting months of observations with string and ceiling-wax equipment at Penrith, NSW, followed by a period of analysis and first attempts at paper writing. The first of four papers, published in 1950, stated "The analysis showed that the spectra of many of the observed bursts conformed to one of three distinct spectral types ... Type I ... Type II ... Type III ..." 
Of the latter I wrote (somewhat tersely I now feel) "the frequency of maximum intensity drifts rapidly (at the rate of the order of 20 (Mc/s) $\sec ^{-1}$ ). towards the lower frequencies. The bandwidth of these bursts at any instant is not usually less than about $50 \mathrm{Mc} / \mathrm{s}$. They last for a few seconds". Each spectral type developed their own personality. On the spectral records type II were the most dramatic, but type III seemed to be the most intellectually challenging because of their extreme simplicity of structure, yet elusive origin. Let me now dwell on them for a while, in the context of Smerd's later involvement.

There were two main problems to be solved in the understanding of type III bursts: (i) what causes the frequency drift? and (ii) what produces the high intensities $\left(\mathrm{T}_{\mathrm{a}} \sim 10^{9} \mathrm{~K}\right)$ ?

As regards (i), Professor J. C. Jaegar of the University of Tasmania (a regular and helpful visitor to Radiophysics) suggested that the frequency drift was due to the differential group retardation of radiation travelling from Sun to Earth. I was able to reject this hypothesis quite unambiguously by an analysis involving the curvature of the ridge-line of the bursts in the time-frequency plane. My analysis showed however that the curvature was consistent with the origin of the frequency drift being caused by an agency travelling through the plasma levels at a ve1ocity of order.3c. My conviction that the drift was indeed due to such a fast agency grew in the 1950's as new evidence accrued from the much broader band spectrograph that we built at the new Dapto field station. The next important evidence was the discovery of first and second harmonics in their spectra and in 1955 I reported to a symposium on solar flares at the I.A.U. General Assembly in Dublin "... harmonic characteristics are observed, suggesting the outward motion of ionized streams exciting the plasma frequencies. The velocities are very high $-3 \times 10^{4}$ to $10^{5} \mathrm{~km} / \mathrm{sec} . "$ Despite the evidence at hand there remained many doubters because such high velocities had not been observed in optical prominences. However three years later we put the matter beyond doubt with the aid of swept-frequency interferometer observations which located the sources of the bursts at solar heights which systematically increased with wavelength. Finally in the late 1960's the type III electrons were directly detected by spacecraft.

\section{Plasma Radiation}

We turn now to the second question: (ii) What produces the high intensities in type III bursts $\left(\mathrm{T}_{\mathrm{a}} \sim 10^{9} \mathrm{~K}\right)$ ? The first real clue emerged from the observation of first and second harmonics which indicated that we were dealing with some kind of resonance radiation. Two possible resonant frequencies presented themselves: the gyro and the plasma frequency. The former was eliminated because theory showed that the fundamental could not escape, and so (as previously quoted) one inferred that the radiation was from excitation of the first and second harmonic of the plasma frequency by the ionized stream. Years earlier D. F. Martyn had suggested plasma oscillations (Langmuir waves) as a possible source of non-thermal solar radio emission but no theory had been developed. V. L. 
Ginsburg and V. V. Zheleznyakov were responsible for the basis of the theory through their remarkable paper of 1959.

The theory of Ginzburg and Zheleznyakov can be represented by a flow diagram (see Figure 6 with dotted line option on right-hand side). The type III electron stream interacting with the background plasma generates Langmuir waves through the process of the two-stream instability. These waves are scattered by thermal ions to generate transverse electromagnetic waves responsible for the type III emission of fundamental frequency. The Langmuir waves also coalesce with thermal Langmuir waves to generate electromagnetic waves at the second harmonic emission.

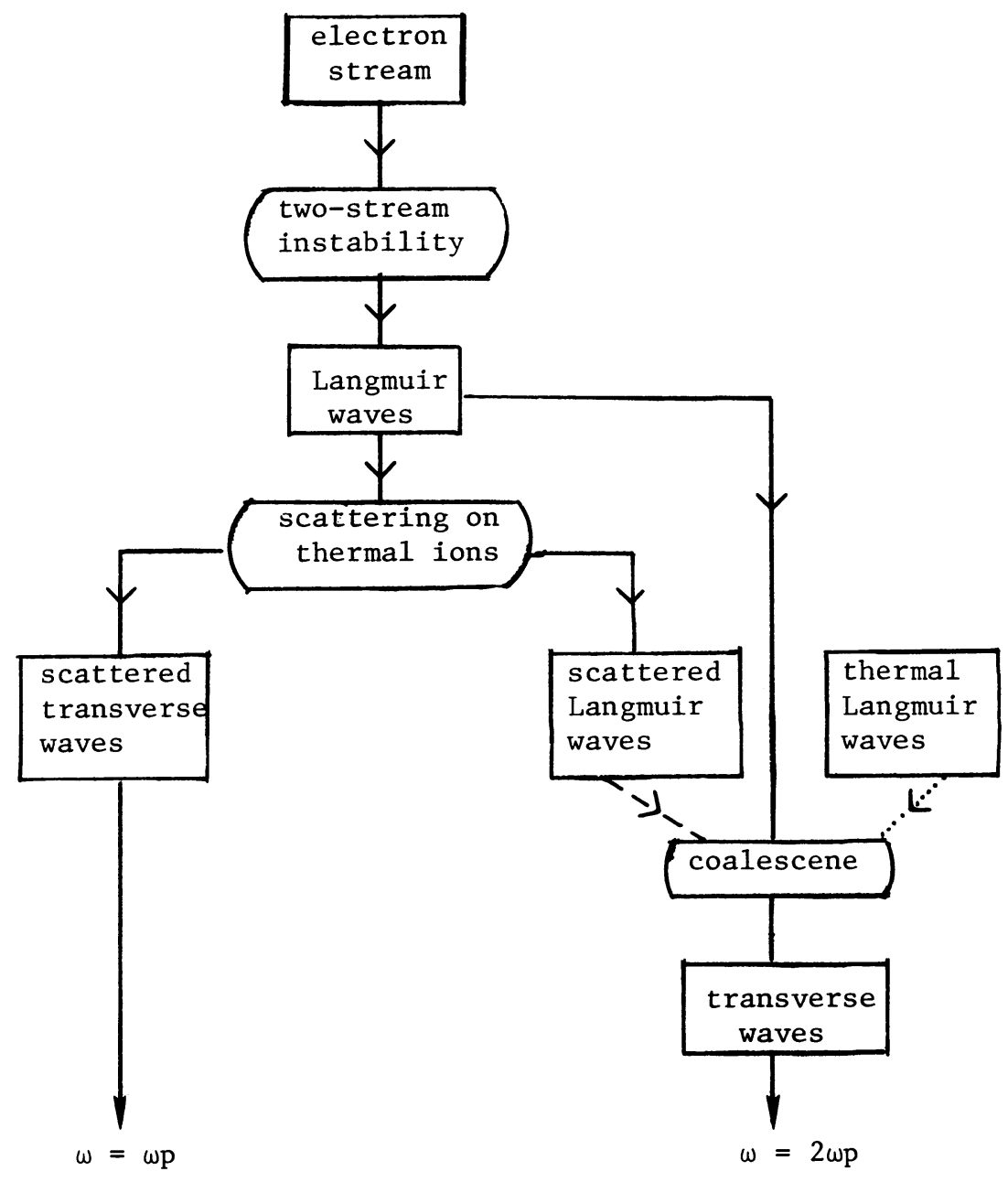

Figure 6. Theory of the generation of first- and second-harmonic radiation from plasma waves excited by an electron stream. The original theory of Ginzburg and Zheleznyakov uses the dotted link, more recent theories the dashed link. 
It is interesting that the theory has largely survived to this day with the modification (dashed-line option in Figure 6) that the generation of second harmonic emission is now seen to be dominated by the result of coalescence between the non-thermal Langmuir waves and their own scattered components. Smerd, through the review of Wild, Smerd and Weiss (1963) did much to promote and popularize this theory, which took some years to penetrate the world of solar radio astronomy. Also Smerd, Wild and Sheridan (1962) made direct use of the theory to explain the anomolous positions on the solar disk of the second harmonic components of type II and type III bursts in terms of emission in the backgward direction.

In recent years the most important question regarding the generation of type III bursts has been the question of how the stream maintains its identity during its outward travel of millions of kilometres and up to tens of seconds duration. In 1964 Sturrock pointed out that the back reaction of the stream to the emission of Langmuir waves should stop the stream in a metre or so. (The problem is relevant to nuclear fusion technology where the lifetime of stable plasma is measured in microseconds).

The solution to this problem is controversial and it is not my intention here to try to be comprehensive. One approach involves the socalled quasi-linear equations first given idependently in 1962 by Vedenov, Velikov and Sagdeev and by Drummond and Pines. Since these equations were first applied by Zheleznyakov and Zaitsev to the theory of type III bursts, contributions have been made by many workers. Smerd followed these developments with characteristic interest and reviewed developments from time to time (e.g. Smerd 1968, Wild and Smerd 1972). But, as so often occurred, his main influence was as a catalyst, this time upon a young colleague $\mathrm{Dr}$. R. Grognard (whose work would, I believe, be better known if his willingness to publish matched his brilliance in that field which lies at the boundary between physics and mathematics). Since Smerd's death Grognard has continued this work, and the remainder of what I now present on this topic is unpublished work of Grognard.

With quasi-linear theory, being a one-dimensional theory, instantaneous plasma phenomena may be graphically represented by a three-dimensional figure in which the $\mathrm{x}$ axis is the one-dimensional spatial axis, the $\mathrm{y}$ axis is $\mathrm{v}=\mathrm{dx} / \mathrm{dt}$ and the $\mathrm{z}$ axis represents an amplitude distribution. We are concerned with two amplitude functions: $f(t, x, v)$, the electron distribution and $D(t, x, v)$, a diffusion coefficient related to the plasmon spectrum, $P=v D$ (Langmuir waves). In normalized units the quasi-linear equations relate $f$ and $D$, thus:-

$$
\begin{aligned}
\frac{\partial f}{\partial t}+v \frac{\partial f}{\partial x} & =\frac{\partial}{\partial v}\left(D \frac{\partial f}{\partial v}+f\right), \\
\frac{\partial D}{\partial t} & =v^{2}\left(D \frac{\partial f}{\partial t}+f\right) .
\end{aligned}
$$

In the right-hand side of the last equation, the first term gives the induced emission, and the second term spontaneous emission of plasmons. 

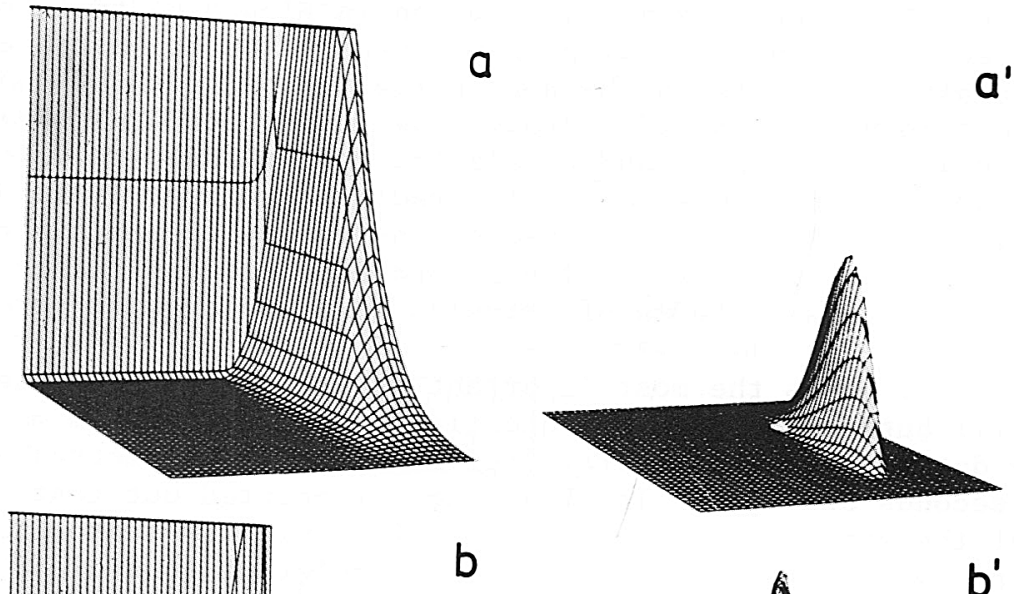

b
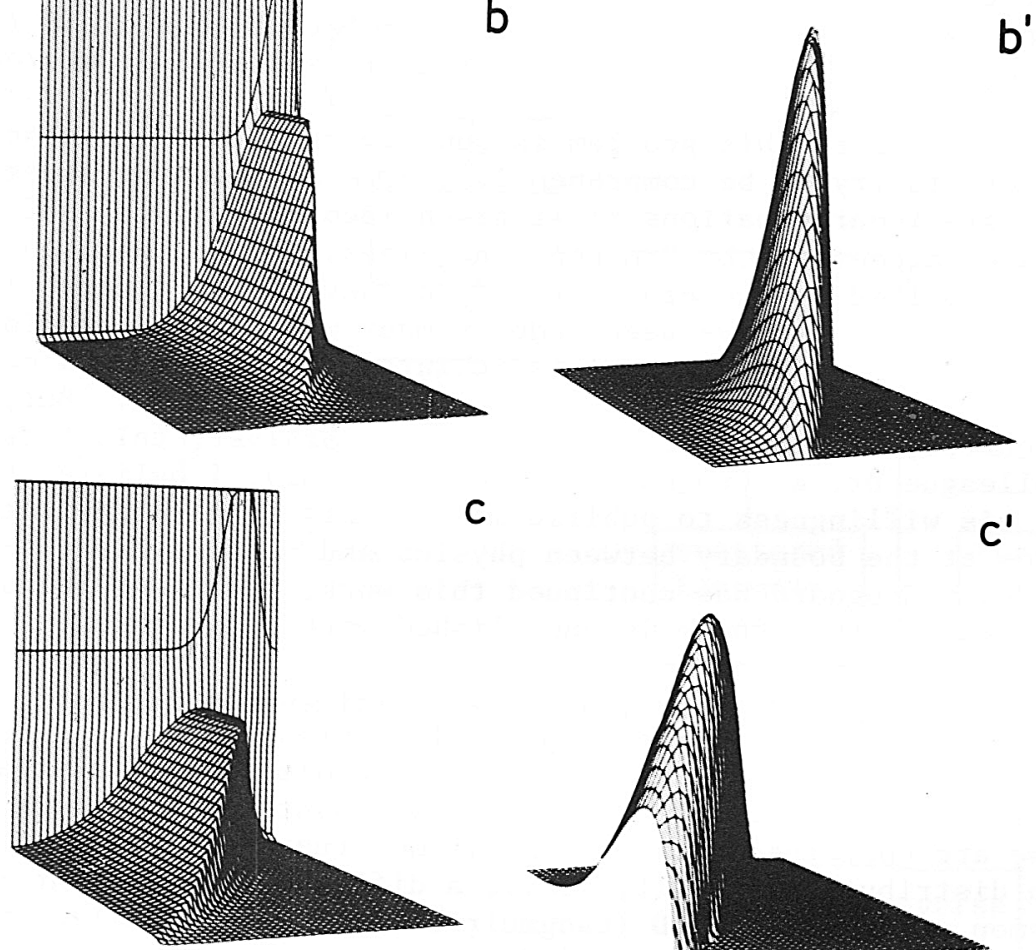

C
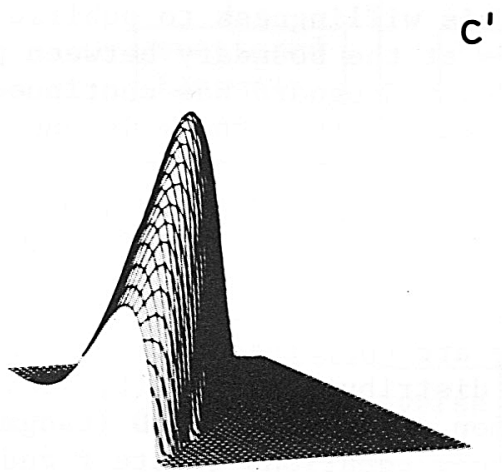

Figure 7. Three frames from moving picture of solution of quasi-linear equations representing the excitation of a type III burst (see text). Electron (left) and plasmon (right) distributions are shown as a function of spatial coordinate $x$ (increasing left to right) and velocity $\mathrm{v}=\mathrm{dx} / \mathrm{dt}$. The 'wall' on the left-hand diagram represents the electron distribution of the background plasma (truncated at 2000 electrons per Debye length). The base measures $2.6 \times 10^{5} \mathrm{~km}$ x 35 thermal velocities. after (a) $0.64 \mathrm{sec;}$ (b) $1.91 \mathrm{sec;}$ (c) 3.18 sec. 
I am assured that the consequences of applying some initial injection of electrons into the system are not at all easy to perceive from these equations. Ryutov and Sagdeev postulated some assumptions which greatly simplified the numberical analysis but Grognard has pointed out some serious deficiencies in their analysis: the only way to be sure of the true consequences of the equations is to solve them numerically and completely. This, for the first time in the form of a moving picture, he has done with the aid of CSIRO's Cyber 7600 (see excerpts in Figure 7). In this case the initial injection of electrons is specified as a boundary condition at $x=0$, such that the electron-density distribution $f(t, o, v)$ corresponds to a Maxwellian distribution of temperature $10^{8} \mathrm{~K}$. The density of electrons rises and falls like the first half of a sine wave enduring for $1 \mathrm{sec}$; the maximum electron density is $10^{3}$ per Debye length; and the background plasma is at $10^{6} \mathrm{~K}$ temperature with electron density corresponding to a plasma frequency of $100 \mathrm{MHz}$.

The results dramatically show that as the electron-density distributions propagates, the position of its leading edge is independent of velocity and the plasmon distribution (corresponding to the radio emission) advances as a well-defined discrete hump. Subject to the inherent limitations of the one-dimensional treatment and also the neglect of plasmon-plasmon collisions, the results give solid support to this interpretation of type III bursts. It also supports the simplifying hypothesis of Ryutov and Sagdeev.

\section{Finale}

In this lecture I have tried to touch on matters which reflected the main intellectual theme of Smerd's scientific life. In so doing I have sampled topics with no attempt at comprehensiveness. I have neglected other important aspects of Smerd's contributions; especially his latter day work on the study in depth of major solar events (e.g. Dulk et al 1976, 1977) in which he showed himself as one who insisted on nosing out all the data: radio, optical EUV, X-ray, corpuscular etc; who insisted on seeing the raw data for himself; and who eventually persevered until he had drained all the physics possible out of the data.

Finally, some of us will remember Steve, outside his work domain, as a person of great sincerity, humility and humour. I believe that you will all agree that he was a tremendous asset at international conferences. But perhaps only those of you who saw him on his home ground saw the complete Steve: with his family; and occasionally, the life and soul of the party, with his guitar as in Figure 8 . How vividly one can remember the accompanying voice that sang:

"He was a bold bad man was that desperado, From Cripple Creek way down in Colorado, And he rode around like a wild tornado, And everywhere he went he gave his war whoop!"

Except that it always came out as Cwipple Cweek ... 


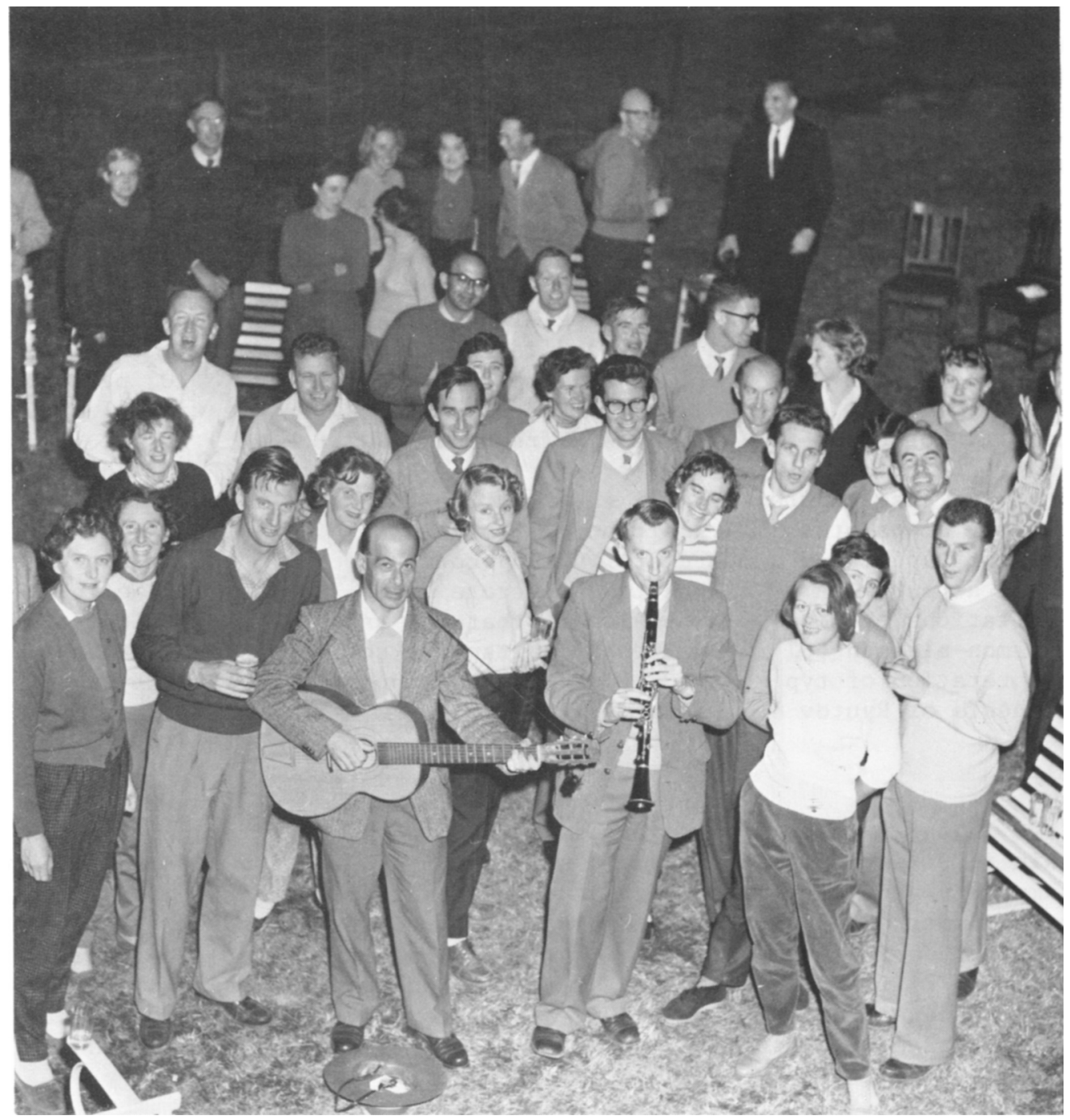

Figure 8. A Dapto party, 3 July 1959 and Smerd the guitarist.

But I leave until the end the one quotation from the culture of our English-1anguage heritage that encapsulates the Stefan Smerd whose self and whose Sun we grew to know and love so well. Oliver Goldsmith has provided the words:-

"For e'en though vanquished, he could argue still;

While words of learned length, and thundering sound, Amazed the gazing rustics ranged around; And still they gazed, and still the wonder grew, That one small head could carry all he knew." 
I am deeply indebted to Drs. R. J. M. Grognard, D. B. Melrose, K. V. Sheridan and G. A. Dulk for assisting in the preparation of this paper.

\section{References}

The list below includes only papers of which S. F. Smerd was an author. References to most other work referred to may be found in the review articles marked thus*.

Dulk, G. A., Smerd, S. F., McQueen, R. M., Gosling, J. T., Magun, A., Stewart, R. T., Sheridan, K. V., Robinson, R. D., and Jaques, S. (1976), Solar Phys. 49, 369.

Dulk, G. A., Sheridan, K. V., Smerd, S. F. and Withbroe, G. L., (1977), Solar Phys. 52, 349.

*Pawsey, J. L. and Smerd, S. F. (1953), "Solar Radio Emission" Chapter 7 of "The Sun" (Ed. G. P. Kuiper, University of Chicago Press).

Smerd, S. F. (1950a), Aust. J. Sci. Res. 3, 34.

Smerd, S. F. (1950b), Proc. I. E. E., London, 97, Pt. III, 447.

*Smerd, S. F. (1968) Proc. A. S. A. 1, 124.

Smerd, S. F. (1970) Proc. A. S. A. 1, 305.

Smerd, S. F. and Westfold, K. C. (1949) Phil. Mag., Series 7, 40, 831.

Smerd, S. F., Wild, J. P. and Sheridan, K. V. (1962), Aust. J. Phys. 15, 180 .

*Wild, J. P. and Smerd, S. F. (1972), Ann. Rev. Astron. \& Astrophys. 10, 159.

*Wild, J. P., Smerd, S. F. and Weiss, A. A. (1963), Ann. Rev. Astron. \& Astrophys. 1, 291.

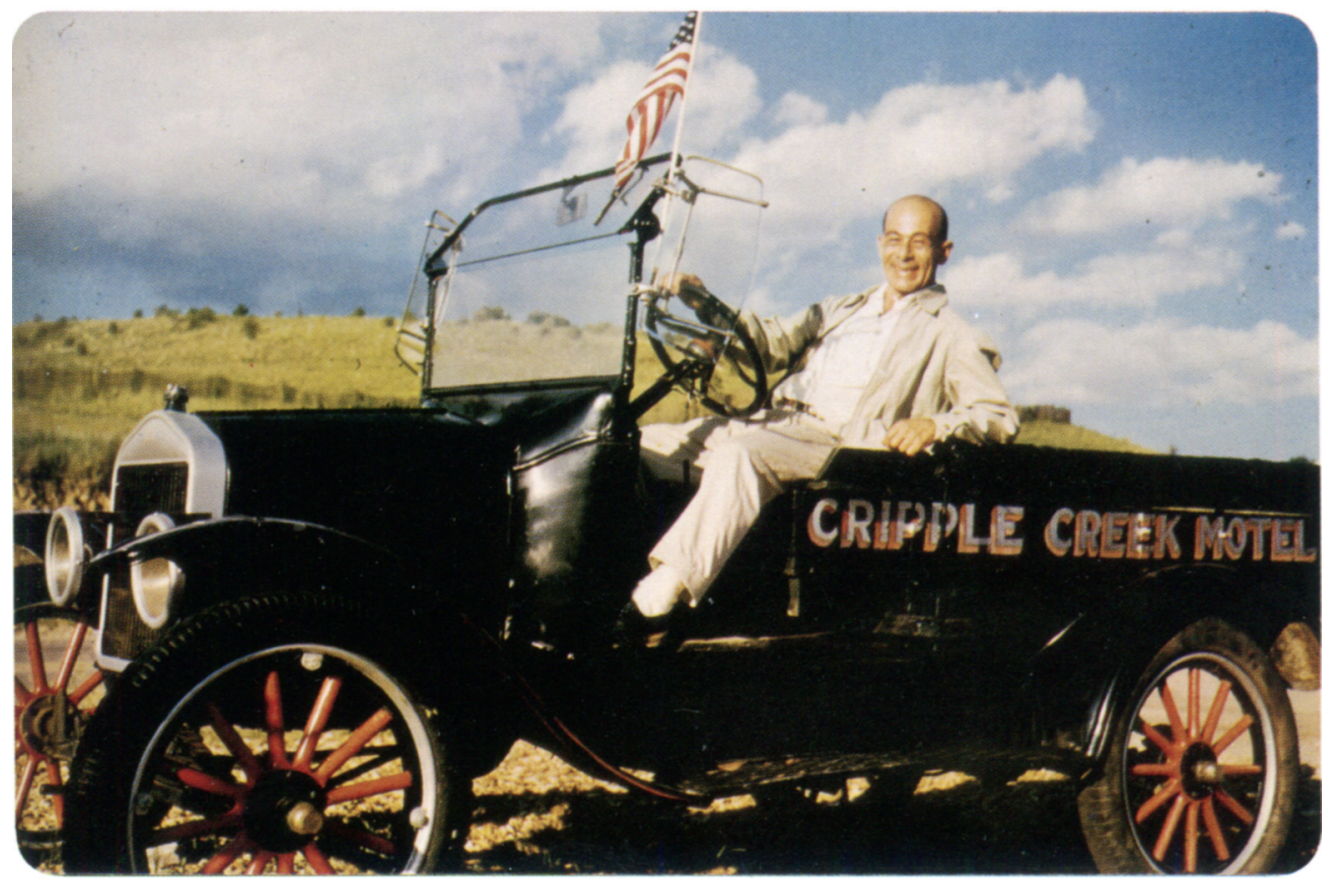

